\title{
Qualitative study of Chinese widows in Hong Kong: Insights for psycho-social care in hospice settings
}

\author{
Amy YM Chow and Cecilia LW Chan, Department of Social Work \& Social \\ Administration, and Centre on Behavioral Health, the University of Hong Kong, \\ Samuel MY Ho, Department of Psychology, the University of Hong Kong, Doris \\ MW Tse, Margaret HP Suen, and Karen FK Yuen, Caritas Medical Centre, \\ Hospital Authority, Hong Kong
}

In an exploratory phenomenological study of seven Chinese widows in Hong Kong, five major themes are identified: relationship with the deceased, relationship with others, grief reactions, coping and emotional expression. This paper is going to focus on first theme: the widows' relationship with the deceased husband along a timeline from the time of diagnosis to after death. The experiences in the final days with the deceased were frequently referred to when the informants shared about the theme. This paper is aimed to give extra voices, grounded from the Chinese widows, to the possible role of hospice and palliative care in affecting the bereavement experience. Issues of awareness and acceptance of death, the supportive environment and support for the family carers, the moment of death, creation of legacies, and sensitivity towards cultural embedded practices are discussed. It is hoped that these voices can help to ignite future research directions for verifying the postulations.

Key words: widows; Chinese; pre-bereavement; bereavement, palliative care

Address for correspondence: Amy Chow, Department of Social Work and Social Administration, the University of Hong Kong, Pokfulam Road, Hong Kong. Email: chowamy@hku.hk 
Qualitative study of Chinese widows in Hong Kong: Insights for psycho-social care in hospice settings

\section{A Introduction}

Palliative care is an approach that "improves the quality of life of patients and their families facing life-threatening illness"1 . The target of care is not limited to patient only, but is extended to their family members as well. The need of family members is not restricted to the time when the patients are still alive. Bereavement care, the care extended to the family members after the death of the loved one, is an integral component of palliative care as well. ${ }^{2-5}$ Bereaved persons are found to be revisiting the memories of the deceased in the final days. Thus, palliative care for the patients and the family members then are inevitably shaped the adjustment in bereavement experience of the family members. ${ }^{6}$ Yet in evaluating the quality of palliative care, assessment are usually obtained from patients ${ }^{7-9}$ and service-providers. ${ }^{10-11}$ Due to ethical and practical considerations, unbiased opinions towards the palliative care from patients and family members prior to the death are difficult to obtain. As suggested by McPherson and Addington-Hall ${ }^{12}$, retrospective or after-death opinion obtained from the proxies, usually the bereaved family members, can be a reliable and feasible alternative. There is a start of expanding the scope of informants on measuring the quality of palliative care to bereaved family members ${ }^{13-15}$, but few was 
found from the Chinese bereaved family members. Kwan ${ }^{16}$ and Li, Chan \& $\mathrm{Lee}^{17}$ has studied on Chinese bereaved family members' experience, but the former is restricted to the participation in last office whereas the latter is limited to the nurse support in Accident and Emergency Department after a sudden death. Martinson and colleagues ${ }^{18-19}$ had other studies, but are also limited to the bereaved parents losing their young children from cancer.

In an exploratory phenomenological study on widows' bereavement experience of losing the husband through chronic illness, the recalled relationship with the deceased emerged as one of the main themes in the analysis of the transcription of the in-depth interview with the widows. The deceased husbands of nearly all the informants were recipients of palliative care before their death. The information provided by the widows can be viewed as an indirect source of information related to perceptions towards the experience in palliative care. The sharing of the informants in the recalled memories with the deceased ranged from the treatment period, the death and the post-death phase. Most of them actually attributed their good adjustment of bereavement to the good relationship with the deceased husband. Since the relationship with the husband between the treatment period and the death moment are inevitably shaped by the experience of receiving palliative care, a lot of postulations 
on the influence of hospice and palliative care on the bereavement adjustment are deduced. Despite of the limitations in generalizing the postulations to a greater population, this study is hoped to add voices of Chinese bereaved persons, who has received palliative care services in Hong Kong, about the perceptions of palliative and hospice care. It is hoped that these voices can help to ignite future research directions for verifying the postulations.

\section{A Hospice services in Hong Kong}

One of the common misconceptions among Hong Kong Chinese people is that hospice is a dumping ground where physicians dispose of "incurable" patients who are left to die in misery. Therefore, there is a generalized reluctance on the part of patients and their family members to consider a referral from oncology, medical or surgical wards to a hospice unit. They see such a referral as a death sentence and feel abandoned by the health care system. The population has very high expectations of the health care system and expects support for their very strong urge to live for as long as possible. In addition many Chinese families object to the breaking of bad news to the patients. ${ }^{20}$ Without the knowledge of the diagnosis and prognosis, the decision to receive hospice care is often delayed or put off completely. 
Nevertheless there seems to be a growing utilization of hospice, and the participants in the research described in this paper provide a personal perspective on its value. Ninety-three percent of the hospital admissions are to public hospitals. Therefore palliative care services develop under the Hospital Authority. There are 249 hospice beds distributed in 10 hospice wards in various hospitals serving the Hong Kong population. There is one free standing hospice in Hong Kong. In addition hospice home care nurses provide domiciliary services in patients' homes. In 2002, among all cancer deaths, $60 \%$ of the patients and their families received hospice palliative care service and they received the care during their last 80-90 days, each patient was admitted on the average of 1.5 admissions for palliative care. The average length of each stay is 16 days. ${ }^{21}$ From Jan to Aug 2004, there were a total of 383 deaths in the hospice service. ${ }^{22}$ The quality of hospice care in Hong Kong is high, staffed by a group of dedicated professionals who are both clinically and emotionally competent to meet the needs of people who are dying. ${ }^{23}$

\section{A Methods}

The study, which originally used to explore the experiences of widows in Hong Kong, 
adopted a qualitative approach in gathering and analyzing the data. The primary data gathering tool was a semi-structured interview with the widowed informant and an interpretive phenomenological analytical approach (IPA) $)^{24-25}$ was adopted. IPA is chosen as it is suitable to explore how individuals perceive particular situations they are facing and how the personal meanings are made. ${ }^{26}$ This matches with our research question of exploring the bereavement experiences of Chinese widows in Hong Kong. IPA concerns with the insiders' world, but also recognizes the dynamic nature of the research process between the informant and researcher. This matches with the authors' beliefs and stances. As suggested by J. A. Smith, ${ }^{24-25}$ an interview schedule with open ended, semi-structured questions was developed. The questions started with the format of "Please share about your ..." In this study the focus was on three issues: 1. the bereavement experience, 2. changes after the death of their spouse and 3. adjustment to bereavement. Procedures in prompting for deeper and wider responses are also included in the interview schedule. The interviews were all audio taped and transcribed for analysis. Ethical approval was obtained from the local research ethics committee. 
B Participants and Procedures:

The participants were recruited from the medical social work department of Caritas

Medical Centre (CMC), a general hospital in Hong Kong, China. The target

participants were widows between 30 and 59 years of age, whose husbands died from

cancer. In one case death followed a chronic illness. They were considered mentally

sound by the referring staff and they were willing to participate in a long interview.

The medical social workers in the medical and hospice wards reviewed the list of their patients who had died within the past three months. They made telephone contact with them, to assess the widows need for services and if none were needed they invited the widows to participate in the study. Once the verbal consent was obtained, information about how to contact the participant was passed on to the researcher (the first author). The participants were contacted via telephone within the first two days after consent was obtained. Through the phone contact, the details and rationale of the study were explained. Seven women agreed to participate. Table 1 contains background information about the participants.

Participants were offered a choice of sites for the interview. These included the medical social work department of the hospital, the University, and the home of the 
participants. Only one of them chose the hospital while the remaining preferred a home visit by the researcher. Upon the interview, participants were briefed again about the information of the study, both verbally and in written form. Interview started after they showed consent to the study by signing the written consent forms. As suggested by Smith, ${ }^{25}$ IPA starts with the detailed transcript of one interview before incorporating the interviews of the other widows into the program. Themes are identified through reading the transcription of the first case a few times. In this study, the process was made easier with the help of the software QSR Nvivo. ${ }^{27}$ The transcription of the interview was stored in softcopy form of rich text. The software systematically stored the transcription of different interviews so that they can be easily retrieved. Despite the soft-ware is designed for English text, it can read Chinese text as well. Thus the transcriptions of the interviews are in Chinese. Through line by line reading, code that embraced the theme of the sentences, or the paragraph can be assigned. The coding process can be done on the screen and the coded text can be retrieved quickly in an organized way. Revisions of the codes can be made easily. Relationships between different codes and data can be shown diagrammatically thus facilitating the process of analysis. ${ }^{28}$ The transcription of the first interview was read three times and codes were assigned to describe the essence of each statement or paragraph offered by the informant. The codes were then thematically aggregated and 
put under a "parent-code". 110 codes were identified after the analysis of the first case. The analysis of the first case was carried out by the first author, then other team members joined in a meeting to code the first case jointly again. There is a great consistency of themes generated in the meeting when compared with those generated by the first author alone. Then the second interview was analyzed solely by the first researcher and 33 new codes were added. Interviews and analyses were carried out simultaneously. A further 41,8 and 5 codes were identified in the $3^{\text {rd }}, 4^{\text {th }}$ and $5^{\text {th }}$ interviews respectively. The transcription of the interview of the sixth and seventh informants yielded no new codes, thus the data collection was stopped as the data is found to be saturated. The process resulted in a total of 197 codes, ranging from different grief reactions to perceptions of relationship with the deceased and the others. The codes were grouped into themes and will be discussed further in the following section of findings.

This sampling method is based on the concept of data saturation as introduced in Grounded Theory Approach. ${ }^{29}$ Instead of having a pre-set sample size of informants, data saturation is the criteria for ending a study. ${ }^{30-31}$

\section{A Results}


Although all the interviews were carried out well after the death of their husband and the leading questions were about their bereavement experiences, these widows were inclined to start the storyline with the time of diagnosis or the time when their husbands were involved in treatment. When they talked about what was helpful, their answers were mainly centered around the conditions and the relationship with the husband at the various points in time before the death, at the time of death and afterwards.

Five major themes were identified: relationship with the deceased; relationship with others; grief reactions; coping; and emotional expression. In this paper, the findings presented relate to the theme focusing on the widow's relationship with the deceased. Themes around their relationship before the death and at the death moment and their ongoing relationship with the deceased were chosen to be included in this paper as these are more relevant to palliative care.

B Before the Death 
C Time to accept and prepare for the death

All these women talked about their appreciation of the time they had to gradually come to terms with the inevitability of the death of their spouse. They described this as a factor which led to better adjustment after the death. The appraisal of the adjustment though is not measured by an object scale, but was rather a reflection of the informant's own perception of their experience.

"Our case is different. If he had died in an accident, I would be extremely disturbed and depressed! But, he was a chronic patient, I was prepared to face it.” (Mrs. D.) "My husband did not die in an accident. He had a chronic illness for a long time... [we witnessed] his deterioration day by day, thus would be able to accept it slowly. More than a year ago, the doctor said that he had no cure. He survived till recently. It was not sudden at all. ” (Mrs. F.)

C Building Fond Memories and Legacies: 
Some informants shared that they adjusted better because of the memories and legacy

left by the deceased husbands.

"He suggested one day, 'Why don't we go to the photo studio to have some family pictures taken. I wanted to leave it to you and our daughters as a memento!' At first I rejected the idea as it cost much. I just told him that we could simply use our own camera... He further insisted and we finally went to the studio to have the photograph taken... The photos now become a truly precious gift to me and our daughters " (Mrs. G)

"This is the special gift from my husband --- a letter that he wrote at his bed side!" (Mrs. C)

"The chaplain suggested that my husband make an audio tape for our daughters. Caritas Medical Center is so thoughtful!” (Mrs. F)

C The physical condition of the patient: a positive image:

These widows vividly remembered the physical appearance of the deceased in the period before he died. Mrs. D attributed her effective coping with her grief to the 
positive physical image of the deceased in her mind in his last moments in life,

"He did not lose much of his weight and he looked well. With my care, he did not have any bedsores and was clean! ... He could still have his hair washed and looked comfortable. It is really a blessing!” (Mrs. D)

C Opportunities were available to provide care for the patients:

The informants talked about having opportunities to provide care for the deceased before the death. These acts were seen as smoothing out the bereavement process.

"No one can take care of him the way I can. I have no regrets, for I have done so much for him!” (Mrs. C).

"I don't have any unfinished business as I have spent so much time with him in the ward!" (Mrs. F) 
"I have to care for both my husband and my family. I did not perform well in supervising my daughter's homework, I just hoped that she was safe at home!" (Mrs. G)

"I was really exhausted. I have to take care of him [the patient], as well as to go to work. More importantly, I have to boil the Chinese herbal medicine for him. It took hours to prepare the medicine!" (Mrs. E)

C The Importance of the Environment of the hospice ward and its services:

Since the hospice ward is the final place of stay of the deceased husbands, most informants paid special attention to the environment of the ward.

“...the hospice ward of CMC [the site of recruitment] is much better than what we had expected. My husband did not want to go there, feeling that he was being transferred to a place to wait for his death... To our surprise, the hospice ward was just like a big, warm family that was so comforting! ... with the sharing with other caregivers and volunteers, I was much relieved. So was my husband...” (Mrs. F) 
“... the environment was decent. The atmosphere was comfortable and relaxing.

Probably the color and lighting of the ward also helped ...” (Mrs. B)

B Moment of Death

C Presence at the moment of death

The presence of the whole family at the moment of death is considered to be very important. The adjustment to bereavement is believed to be better when they have done so.

"The hospital staff are very nice. They called me when my husband was in a critical condition. I would blame myself if I could not be by his side (during the moment of his last breath)!" (Mrs. E)

"It was really my blessing to be able to accompany him during his departure. The nurse was quick to notify me that he was dying! If they didn't, then I might have missed the moment and would have regretted it for a whole life time." (Mrs. C)

"I think it is a blessing, for me to have witnessed his death, with him dying in my 
arms. I was fearful at that time, just hoping that someone would come to me and confirm whether he was really dead. The nurse was quick and she told me that he has gone." (Mrs. C).

C Looking Peaceful at the moment of death:

It means a lot to the widow if her husband looked peaceful at the moment of death. "He looked so peaceful, and without any complaints of pain! He looked as if he was sleeping peacefully!" (Mrs. F)

C Emotional containment at the death moment:

In our sample of widowed informants, a number of them described themselves as trying not to cry and to contain their emotions. They seemed to be proud of what they had achieved at that period of time.

"[I didn't cry] ... for I feared that it would affect his path to the new world (reincarnation). I really hoped that he wouldn't be worrying about me!" (Mrs. A)

"The day before he died, he told me that he was leaving. I even asked him to go 
without worrying about us. I tried my best to make him leave at ease!" (Mrs. A)

\section{A Discussions}

Witnessing the deterioration of health and the dying process of the family members is a difficult experience. This experience is imprinted onto the mind of the family members in their rest of their life, and vividly surfaced into their consciousness from time to time during the bereavement process. In contrary, memories of pleasant experience with the deceased or fulfilled experience in the end-of-life care can be soothing the pain in the bereavement experience. Based on the findings in this part of the study, five postulations in palliative care are integrated. They are awareness and acceptance of the death, supportive environment and support to family carers, positive memory of pleasant experiences with the deceased especially at the moment of death, creation of legacies, and sensitivity towards cultural embedded practice.

\section{B Awareness and acceptance of death}

From the perspective of the widows, losing their beloved husbands through this slow deterioration process associated with a long illness was more bearable when they compared their experience with those who had lost their husbands from a sudden death such as an accident or suicide. They felt they had more time and space to digest 
the bad news. Thus it is hypothesized that if the patients and families have an open awareness of the impending death, the bereavement adjustment will be better. The open awareness has to be achieved when both parties have the information of the diagnosis and prognosis.

Breaking of bad news has been a controversial topic for Chinese families. ${ }^{20}$ On the one hand, the patient might request the doctor to withhold disclosure of their diagnosis of having a terminal illness from their family members. On the other hand, the request might also be initiated by the family members, who do not want the patient to be told the truth. ${ }^{6}$ Each claims that the underlying reason for such a request is to provide protection and care for the other family members. In contrast, Fielding in a survey of 1136 general households in Hong Kong, reported that 95\% of the respondents indicated that they would have liked to have known the diagnosis even if it was a bad one. This is an important finding. The same percentage of respondents further indicated that they did not want their diagnosis to be shared with family members. ${ }^{32}$ Fielding interviewed a patient sample. He asked them to recall their reactions to receiving the bad news. Approximately $25 \%$ considered themselves as depressed whereas the remaining perceived themselves as calm. ${ }^{33}$ In a qualitative study carried out by Mak, ${ }^{34-35}$ awareness of dying was identified by the Chinese 
hospice patients as the foremost essential element of a good death. With the information of the prognosis and openness of communication, the family could plan realistically on how to make use of the remaining precious moments together. Our data is consistent with these findings. In contrast to traditional beliefs, talking about death did not hasten its coming .Instead our informants pointed to the positive outcomes of their open communication with their husbands while he was dying. The studies of the effect of open communication with the dying on bereavement adjustment will be a good research topic in Chinese culture.

B The supportive environment and support for family carers

Our findings also reflect the value of the hospice experience for these widows. Unlike a regular acute hospital ward, the CMC hospice ward was equipped with a sofa, family corner, a pantry with a refrigerator and microwave oven where food can be stored or heated. There are also volunteers who run a snack bar, and group activities in the ward. Patients and family members are very sensitive to the clues of the ward environment during the last days of the patient's life. The decorations on the walls, color of the curtains and bed-sheets were highly appreciated. The environment was homely, warm and welcoming. The individualized care and concern by the staff and 
volunteers in the hospice ward had made their stay very special. The widows perceived that a decent place to leave the world as a last respect to the deceased husband. At first, they worried that hospice was a dumping ground, and sending their husband to hospice might be a regretful decision. The good environment disputed all the unnecessary worries.

The good environment is confined to the hardware but also the software. Informants expressed appreciation to the dedication, care, commitment and the warm facial expressions of the hospice staff. Though family is the unit of care in hospice belief, the informant preferred to have the chance to care for their dying husbands. In our previous research we observed that the grief of a Chinese person can be characterized as other-focused grieving ${ }^{36}$ emphasizing the interdependent -relational dimensions of grief. ${ }^{37}$ Family members place greater concern on the well-being of their dying loved ones rather than on themselves. They shared that their involvement in caring for their husbands in the hospice ward, though stressful, did have a positive impact on their bereavement experience. They were consoled in that they had done everything they could. Haley ${ }^{38}$ echoed the same theme and suggested that satisfaction and a greater sense of self-worth might be the rewards of caring for dying patients. He suggested that the stress of care giving can be lessened by psychological support, practical 
assistance and education as well as advice from healthcare professionals. Similarly, another qualitative study of Hong Kong families also found that family caregivers regarded the tough care-giving tasks as meaningful. ${ }^{39}$ The care is perceived as a sign of commitment, an act of love, and determination. The caregiver can also find meaning in life and peace of mind. A longitudinal study of the bereaved family caregiver in Italy found that the 1-year post death maladjustment was correlated with the problem in care-giving. ${ }^{40}$ Thus supporting the family caregiver in care-giving might create a positive impact on family adjustment to the bereavement process. This is very consistent with Chinese traditional thinking. At the same time, we can hypothesize that perceived difficulties in caring for the patient might be an early alarm for the need of bereavement support after the death of the patients. We need to keep in mind that family caregivers need to be cared for and at the same time that they are offered opportunities and support to provide care for their dying family member.

B The moment of death

In Chinese culture, it is seen as a curse when family members are not present. Sons, in particular, should witness the death, otherwise reincarnation might be less smooth. The widows reported that they felt good about being able to be around when their 
husbands died. They had also tried their best to arrange that other family members, especially their sons, share this special moment with the dying patient.

Since it means so much to the widows that they witness the moment of their husband's death, it is crucial that hospices make provision for the family to stay with the terminal patients at the end of his life.

The peaceful environment surrounding the time of death was the most frequently remembered episode in the widows' narratives. If space can be provided for the family after the death of their loved ones within the hospice, the family can then express their emotions freely and spend time with the body of the deceased, giving them a sense of completion.

Besides, the physical state of patient at the death moment is considered as an important factor. Freedom from pain and dying with dignity are regarded as the most important aspects of a good death in a general population survey in Hong Kong. ${ }^{41}$ Chao ${ }^{42}$ identified 3 components of good dying as perceived by Chinese terminally ill patients : well-being of body, mind and spirit. Well-being of the physical body is not only crucial to the patients but also to the family members who spent long hours by 
the bedside to provide care. These memories of how the patient looked stayed vividly in the minds of the widow for a long time.

The quality of pain and symptom control can, for the most part guarantee that the patients die in peace and with little discomfort. Thus, if possible, given the nature of their illness, the appearance and physical condition at the moment of death should be attended to. Allowing sufficient time for the family to notify all their family members to come is important, thus minimizing any discomfort they may feel for not fulfilling this obligation.

B Creation of Legacies

$\mathrm{Attig}^{43}$ noted the importance of a legacy from the deceased. A legacy is something handed down from an ancestor or predecessor, and can be crucial to the grieving process. The legacy can be biological (inheriting the physical features or personalities), materialistic (inheriting the valuables and things like writing, photographs) or spiritual (the teaching, the dreams and ambitions). As the informants in our study are all spouses, and therefore not related to the deceased by blood, they focused on the materialistic and spiritual legacies of their deceased husband. The 
materialistic legacies need not be something with monetary value, but are usually

invaluable from a personal point of view. Worden ${ }^{44}$ found the bereaved holding onto objects that belonged to the deceased as one of the normal responses to the death.

They can be seen as symbolic linking objects connecting them to the deceased. In our study, the linking objects ranged from family photographs, audiotape, gifts, paintings and letters. Actually, these linking objects can be produced while in hospice and palliative care. The staff often facilitates this kind of activity. ${ }^{45}$

B Sensitivity towards Cultural Embedded Practice

Death is a psychosocial and spiritual event that is inevitably culture-laden. Through history rituals are developed around the death and dying process. Many of these rituals are not taught in textbooks or in formal schooling but are usually passed on through observation and word of mouth. The Chinese have a rich culture that provides many rituals around death and dying. ${ }^{46}$ As shared by the informants, the importance of being present at the death moment, the peaceful look of the deceased with his eyes and mouth closed, as well as the suppression of emotions by the bereaved at the moment of the death are all cultural prescriptions of a good death for the deceased. In the philosophy of Chinese Medicine, either excessive or the absence of emotions are 
seen as related to physical problems. ${ }^{47}$ In order to maintain a psychological homeostasis or equilibrium, the Chinese tend to control the intense expression of emotions. In facing the intensive emotional experience of the death of a loved one, the Chinese still use emotional control as a coping strategy. Another reason for this is rooted in the Buddhist belief --- the spirit of the deceased would linger after the death for the first few days, and the transitions to the afterlife would be interrupted if the family members were very emotional. Thus it is not uncommon to have Chinese families appeared to be apathetic and calm around the death bed. Instead of viewing them to have masked grief or absent grief, the suppression of emotions does have a cultural purpose. Thus as a hospice care worker, these are important criteria to be watched for during the provision of care.

\section{A Conclusion}

The study is one aimed at understanding the personal perception of the widows in the relationships with deceased. From the thread of the relationship with the deceased as described by the bereaved, insights are gained for practices in hospice and palliative care. This is an exploratory study, and therefore the findings cannot be over-generalized. These findings provide the basis for further research into a very 
complex field.

We close with a note about the value of the study itself to the bereaved. In contrast to expectations they were not reluctant to participate in this research. The informants were open to share their experiences. All of them felt good about the process. They felt a sense of comfort because someone was willing to listen to the stories that they had kept to themselves for a long time. They also treasured the opportunity to contribute to the development of hospice and palliative care services that they themselves had benefited from.

\section{Acknowledgement}

The work described in this article was partially supported by a grant from the Research Grants Council of the Hong Kong Special Administrative Region, China (Project No. HKU 7110/01H). The authors would like to extend their gratitude to Prof. Phyllis Silverman and Dr. Sam Silverman for their valuable feedback to earlier drafts of this paper. Part of the content of this paper has been presented at the $10^{\text {th }}$ Hong Kong International Cancer Congress, on November 19, 2003, Hong Kong SAR, China

\section{References}

1. World Health Organization, WHO. [homepage on internet]. Geneva: World Health Organization; 2006 [cited March 15, 2006]. Palliative Care [first screen]. Available from: http://www.who.int/cancer/palliative/en/ 
2. Parkes CM. Bereavement. In: Doyle D, Hanks GWC, MacDonald N, editors.

Oxford textbook of palliative medicine. 2nd ed. Oxford ; New York: Oxford

University Press; 1998. p.995-1010.

3. Kissane DW. Bereavement. In: Doyle D, Hanks GWC, MacDonald N, editors.

Oxford textbook of palliative medicine. 3rd ed. Oxford ; New York: Oxford University

Press; 2004. p.1135-53.

4. Payne S, Relf M. The assessment of need for bereavement follow-up in palliative and hospice care. Palliat Med. 1994;8(4):291-7.

5. Payne S. The role of volunteers in hospice bereavement support in New Zealand.

Palliat Med. 2001;15(2):107-15.

6. Chan KS, Lam ZCL, Chun RPK, Dai DLK, Leung ACT. Chinese patients with terminal cancer. In: Doyle D, Hanks GWC, MacDonald N, editors. Oxford textbook of palliative medicine. 2nd ed. Oxford ; New York: Oxford University Press, 1998: p. 793-795.

7. Sulmasy DP, McIlvane JM. Patients' ratings of quality and satisfaction with care at the end of life. Arch Intern Med 2002;162(18):2098-104.

8. Sulmasy DP, McIlvane JM, Pasley PM, Rahn M. A scale for measuring patient perceptions of the quality of end-of-life care and satisfaction with treatment: the reliability and validity of QUEST. J Pain Symptom Manage. 2002;23(6):458-70. 
9. Sahlberg-Blom E, Ternestedt BM, Johansson JE. Is good 'quality of life' possible at the end of life? An explorative study of the experiences of a group of cancer patients in two different care cultures. J Clin Nurs. 2001;10(4):550-62.

10. Brazil K, McAiney C, Caron-O'Brien M, Kelley ML, O'Krafka P, Sturdy-Smith C. Quality end-of-life care in long-term care facilities: service providers' perspective. $J$ Palliat Care. 2004;20(2):85-92.

11. Thompson G. Determining the process of providing quality end-of-life care to hospitalized adult medical patients: the perspectives of nurses. Can Oncol Nurs J. 2003;13(2):131-2.

12. McPherson CJ, Addington-Hall JM. Judging the quality of care at the end of life: can proxies provide reliable information? Soc Sci Med .2003;56(1):95-109.

13. Curtis JR, Patrick DL, Engelberg RA, Norris K, Asp C, Byock I. A measure of the quality of dying and death. Initial validation using after-death interviews with family members. J Pain Symptom Manage 2002;24(1):17-31.

14. McPherson CJ, Addington-Hall JM. Evaluating palliative care: bereaved family members' evaluations of patients' pain, anxiety and depression. J Pain Symptom Manage 2004;28(2):104-14.

15. Morita T, Hirai K, Sakaguchi Y, Maeyama E, Tsuneto S, Shima Y. Measuring the quality of structure and process in end-of-life care from the bereaved family 
perspective. J Pain Symptom Manage 2004;27(6):492-501.

16. Kwan C. Families' experiences of the last office of deceased family members in the hospice setting. Int J Palliat Nurs 2002;8(6):266-75.

17. Li SP, Chan CW, Lee DT. Helpfulness of nursing actions to suddenly bereaved family members in an accident and emergency setting in Hong Kong. J Adv Nurs 2002;40(2):170-80.

18. Martinson IM, Zhong BH, Liang YH. The reaction of Chinese parents to a terminally ill child with cancer. Cancer Nurs 1994;17(1):72-6.

19. Papadatou D, Martinson IM, Chung PM. Caring for dying children: a comparative study of nurses' experiences in Greece and Hong Kong. Cancer Nurs 2001;24(5):402-12.

20. Tse CY, Chong A, Fok SY. Breaking bad news: a Chinese perspective. Palliat Med 2003;17(4):339-43.

21. Chan KS, Siu Y, Leong CH. Development of hospice palliative care in Hong Kong. Proceedings of the 5th Asia Pacific Hospice Conference; 2003 March 5-8; Osaka, Japan.

22. Chan CLW. Personal communication with Dr. Michael Sham, Chairperson, Co-ordinating Committee of Hospice Care, Hospital Authority, Hong Kong on Nov 2, 2004, 2004. 
23. Fielding R, Chan CLW. Psychosocial oncology \& palliative care in Hong Kong : the first decade. Hong Kong: Hong Kong University Press, 2000.

24. Smith JA. Semi-structured interviewing and qualitative analysis. In: Smith JA, Harre R, Langenhove LV, editors. Rethinking Methods in Psychology. London: Sage, 1995:9-26.

25. Smith JA, Jarman M, Osborn M. Doing interpretative phenomenological analysis. In: Murray M, Chamberlain K, editors. Qualitative Health in Psychology : Theories and Methods. London: SAGE, 1999:218-240.

26. Smith JA, Osborn M. Interpretative phenomenological analysis. In Smith JA, editor. Qualitative Psychology: A Practical Guide to Research Methods. London: SAGE, 2003:51-80.

27. Qualitative Solutions \& Research Pty. Ltd. NVivo 2.0. Melbourne, Vic.: QSR International Pty Ltd., 2002.

28. Lee RM, Fielding NG. Tools for qualitative data analysis. In: Hardy MA, Bryman A, editors. Handbook of data analysis. London: SAGE, 2004:529-546.

29. Glaser BG \& Strauss AL. The discovery of grounded theory: strategies for qualitative research. London: Weidenfeld and Nicolson, 1968.

30. Charmaz K. Grounded theory: Objective and constructivist methods. In Denzin NK \& Lincoln YS, editors. Handbook of Qualitative Research. Thousand Oaks, CA: 
SAGE, 2000, 509-535

31. Morse JM. The significance of saturation. Qualitative Health Research; 1995, 5:

$147-149$

32. Fielding R, Hung J. Preferences for information and involvement in decisions during cancer care among a Hong Kong Chinese population. Psycho-Oncology 1996;5(4):321-29.

33. Fielding R, Wong L, Ko L. Strategies of information disclosure to Chinese cancer patients in an Asian community. Psychooncology 1998;7(3):240-51.

34. Mak MH. Awareness of dying: an experience of Chinese patients with terminal cancer. Omega (Westport) 2001;43(3):259-79.

35. Mak MH. Accepting the timing of one's death: An experience of Chinese hospice patients. Omega (Westport) 2002;45(3):245-60.

36. Tsui YKY. Other-focused grief. 8th Hong Kong International Cancer Congress. Hong Kong, 2001.

37. Ho SMY, Tsui YKY. Interdependence in death and grief among Hong Kong Chinese. Newsletter of Hong Kong Society of Palliative Medicine 2002;2002(1). 38. Haley WE. Family caregivers of elderly patients with cancer: understanding and minimizing the burden of care. J Support Oncol 2003;1(4 Suppl 2):25-9.

39. Mok E, Chan F, Chan V, Yeung E. Family experience caring for terminally ill 
patients with cancer in Hong Kong. Cancer Nurs 2003;26(4):267-75.

40. Rossi Ferrario S, Cardillo V, Vicario F, Balzarini E, Zotti AM. Advanced cancer at home: caregiving and bereavement. Palliat Med 2004;18(2):129-36

41. Chan WCH, Chan HY, Chan FMY. Perceptions of Good Death: How does it affect end-of-life care decisions? Proceedings of the 11th Hong Kong International Cancer Congress; 2004 November 10-12; Hong Kong, China.

42. Chao C.The meaning of good dying of Chinese terminally ill cancer patients in Taiwan. Proceedings of the 3rd Asia Pacific Hospice Conference; 1999 June 3-5, Hong Kong, China.

43.Attig T. The heart of grief : death and the search for lasting love. New York ; Oxford: Oxford University Press, 2000.

44. Worden JW. Grief counseling and grief therapy : a handbook for the mental health practitioner. 3rd ed. New York: Springer Pub., 2002.

45. Chow AYM, Koo BWS, Koo EWK, Lam AYY. Turning grief into good separation: Bereavement services in Hong Kong. In: Fielding R, Chan CLW, editors. Psychosocial oncology \& palliative care in Hong Kong : the first decade. Hong Kong: Hong Kong University Press, 2000:233-54

46. Chan CLW. Death awareness and palliative care. In: Fielding R, Chan CLW, editors. Psychosocial oncology \& palliative care in Hong Kong : the first decade. 
Hong Kong: Hong Kong University Press, 2000:213-32.

47. Chan CLW. An Eastern body-mind-spirit approach: A training manual with one-second techniques. Hong Kong: Department of Social Work\& Social Administration, the University of Hong Kong, 2001 
Table 1 : Demographic information of the participants

\begin{tabular}{llllll}
\hline Code & $\begin{array}{l}\text { Age of } \\
\text { participants }\end{array}$ & $\begin{array}{l}\text { Age of husband } \\
\text { when died }\end{array}$ & Illness of Husband & $\begin{array}{l}\text { Months } \\
\text { after death }\end{array}$ & Place of interview \\
\hline A & 38 & 42 & Renal Failure & 4 & Participant's home \\
B & 44 & 53 & Cancer & 11 & Participant's home \\
C & 58 & 61 & Cancer & 5 & MSW Office \\
D & 38 & 40 & Cancer & 4 & Participant's home \\
E & 52 & 52 & Cancer & 5 & Participant's home \\
F & 32 & 43 & Cancer & 4 & Participant's home \\
G & 40 & 49 & Cancer & 2 & Participant's home \\
\hline
\end{tabular}

